[Aus dem hygienischen Institut der Universität Königsberg.]

Director: Prof. Dr. von Esmarch.

\title{
Bakteriologische Erfahrungen über die Königsberger Thierlymphe.
}

\author{
Von \\ Kgl. Stadtwundarzt Dr. Ascher, \\ Assistenten des Lymphinstitutes. \\ und \\ Dr. Symanski, \\ I. Assistenten des hygievisehen Institutes.
}

In dem „Bericht über die Thätigkeit der von dem Hrn. Minister der geistlichen, Unterrichts- und Medicinal-Angelegenheiten eingesetzten Commission zur Prüfung der Impfstofffrage " hatte der Berichterstatter Frosch festgestellt, dass in den zur Prüfung übersandten zahlreichen Impfproben aus den acht preussischen Impfanstalten keinerlei für den Menschen pathogene Keime vorkamen, und dass den fünf gefundenen, für Thiere pathogenen Staphylococcus-Arten, wie Impfversuche an Kindern gezeigt hatten, keine Pathogenität für Menschen zuzuschreiben sei. Keimzählungen hatten ferner die schon früher von Schulz-Berlin festgestellte Thatsache bestätigt, dass mit dem zunehmenden Alter der mit Glycerin versetzten Kälberlymphe die Keimzahl ganz beträchtlich und zwar im Verhältniss zum Glycerinzusatz abnahm. Versuche, eine ganz keimfreie und doch praktisch brauchbare Lymphe zu gewinnen, waren fehlgeschlagen; es hatte sich aber auch gezeigt, dass die Keimzahl der Lymphe auf die Reizerscheinungen beim Kinderarm keinen Einfluss hat. Vielmehr hatte Freyer-Stettin gezeigt, dass die Reizerscheinungen individueller Natur seien, da dieselbe Lymphe bei verschiedenen Kindern wechselnde Reactionen hervorrief, während dasselbe Kind, das auf seinen beiden Armen mit verschiedener Lymphe gleichzeitig geimpft war, gleichmässig mit positiver

${ }^{1}$ Berlin, Julius Springer. 1896. 
oder negativer Reaction antwortete. Es konnte ferner festgestellt werden, dass eine Verdünnung der Lymphe und eine möglichst weite Entfernung der einzelnen Impfpusteln von einander die Reizerscheinungen wesentlich verminderte. Die obere Grenze des Verdünnungsgrades für eine praktiseh brauchbare Lymphe scheint eine zehnfache $z \mathfrak{u}$ sein. Eine ganz reizlose Lymphe zu erzielen erwies sich unmöglich. Controluntersuchungen der von Dr. Landmann hergestellten keimfreien Lymphe, deren Herstellungsart nicht bekannt, deren Wirkung aber eine abgeschwächte war, ergaben keine Minderung der Reizerscheinung gegenüber keimhaltiger Lymphe, die auf den anderen Arm desselben Kindes verimpft war. Etwaige Reizerscheinungen traten stets gleichmässig auf beiden Armen desselben Kindes, das auf dem einen Arme mit Landmann'scher, auf dem anderen mit Institutslymphe geimpft war, auf, erwiesen sich also als von der Individualität des Kindes abhängig. Die Untersuchung gerade dieser, bei MerckDarmstadt hergestellten Lymphe wurde hervorgehoben, weil La nd mann auf der 67. Versammlung deutscher Naturforscher und Aerzte in Lübeek ${ }^{1}$ das Vorkommen von virulenten Streptokokken in der Lymphe und die Abhängigkeit der Reizerscheinungen von diesen behauptet und später ${ }^{2}$ mitgetheilt hatte, dass er im Besitze einer keimfreien und doch wirksamen Lymphe sei.

Die Resultate der Commission betr. die Zahl und die Virulenz der Keime, der Unabhängigkeit der Reizerscheinungen vou diesen, der Abnahme nach Zeit und Verdünnungsgrad konnten von Kirchner für die Lymphe von 18 Kälbern der Impfanstalt Hannover ${ }^{3}$ bestätigt werden, eine Untersuchung, die deshalb besonders werthvoll war, weil sie alle Proben einer Anstalt umfasste. Zu demselben Resultate kam auch Dreyer, ${ }^{4}$ der die Lymphe der staatlichen Impfanstalt zu Darmstadt untersuchte und zwar in zwei Jahren: 1896 und 1897. In beiden Jahren erhielt er dasselbe Resultat. Zwei Mal hatten seine Thierversuche ein positives Resultat, indem zwei Mäuse, die eine bei subcutaner, die andere bei intraperitonealer Impfung eingingen. Culturell konnten Streptokokken nachgewiesen werden, die aber nach ihrer Isolirung weder für Mäuse noch für Menschen virulent waren. Dreyer machte auch mit den von ihm reingezüchteten Streptokokken, weissen, grauweissen und gelben, Impfversuche an Menschen und Mäusen, die uns jedoch für die praktischen Schlussfolgerungen weniger wichtig erscheinen als die Versuche mit der Verimpfung der Lymphe, zumal sich keine Correspondenz zwischen den Er-

'Hygienische Rundschau. 1895. V. Jahrg. S. 975.

${ }^{2}$ Ueber die animale Lymphe. Ebenda. 1896. VI. Jahrg. S. 441.

${ }^{3}$ Ueber den Keimgehalt animaler Lymmphe. Diese Zeitschrift. 1897. Bd. XXIV.

4 Bakteriol. Untersuchungen v. Thierlymphe. Diese Zeitschrift. 1898. Bd. XXVII. 
gebnissen mit der Verimpfung der Lymphe und der in ihr enthaltenen, nachträglich isolirten Keime herausstellte. Es sei noch bemerkt, dass die Verimpfung der aus der Lymphe gezüchteten Reinculturen auf den menschlichen Arm, die Dreyer an sich anstellte, nur geringe entzündliche Erscheinungen hervorrief; dies galt sowohl für Staphylo- wie Streptokokken. Dreyer macht selbst darauf aufmerksam, dass die Reactionen auf die Verimpfung von Reinculturen keine Bedeutung für die Verimpfung von Lymphe, die derartige Keime enthält, haben schon wegen der Menge der verimpften Keime.

Wir haben uns deshalb in unseren Versuchen auf die Verimpfung der gesammten Lymphe beschränkt.

Wir hatten zunächst die Absicht, ebenfalls die Abnahme der Kreimzabl und die Virulenz nicht der Keime, sondern, was ja praktisch wichtiger ist, der gesammten zur Verwendung kommenden Lymphe zu untersuchen. Von der Untersuchung der Abnahme der Keimzahl mussten wir jedoch Abstand nehmen; bei den hiesigen klimatischen Verhältnissen kamn erst Ende März mit der Impfung der Kälber begonnen werden, und da die weitaus meiste Lymphe im Mai gebraucht wird, gelangt sie so jung zur Verwendung, dass von einer wesentlichen Abmabme der Zahl der Keime bei dem nicht sehr grossen, hier gebräuchlichen Verdünnungsgrad $1: 4$ Glycerinwasser $(3: 1)$ - nicht die Rede sein kann. Untersuchungen der Abnahme der Keimzahl konnten nur in zwei praktischen und in zwei Laboratoriumsversuchen vorgenommen werden. Das erste Mal handelte es sich um eine Lymphe, die an dem Tage ibrer Bereitung, den 29. IV. 1898, untersucht und später zu Versuchen an Kindern benutzt war (18. V.), die aber eine gewisse Abnahme der Keimzahl von $\infty$ auf 1184500 gezeigt hatte. - In praxi wurde die Lymphe stets kurze Zeit nach ihrer Bereitung zu Impfungen an Kindern und zwar zunächst zu Probeimpfungen an je 6 Impflingen und 6 Wiederimpflingen in der hiesigen Lymphgewinnungsanstalt benutzt. Sie ergab stets gute Erfolge sowohl hinsichtlich des personellen wie des Schnitterfolges. Stärkere Reizerscheinungen fehlten stets. Nur einmal - Kalb 12/14 - batten sich bei der Impfung am 19. IV. bezw. der Besiehtigung am 26. IV. bei den Wiederimpflingen, nicht aber bei den Erstimpflingen etwas heftigere Reactionen, die in stärkerer Röthung des Oberarmes bestanden, gezeigt. Die Untersuchung ergab 7024000 Keime pro Cubikcentimeter Lymphe, jedoch keine Virulenz für Thiere, wie Impfversuche an 2 granen und 1 weissen Maus zeigten. A.uch fehlten hier, wie bei allen unseren Untersuchungen, Streptokokien.

Unsere Untersuchungsmethode war der von Dreyer ${ }^{1}$ beschriebenen

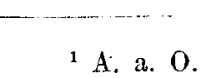


ähnlich. Zum Plattenuntersuchen auf Keimzahl wurde mit steriler Pipette $0 \cdot 1^{\mathrm{ecm}}$ Lymphe entnommen, in $2 \cdot 5^{\mathrm{cm}}$ steriler Bouillon vertheilt; von diesem Gemisch wurde $0.1 \mathrm{~cm}$ entnommen und mit $10^{\mathrm{ccm}}$ Glycerinagar (möglichst frisch bereitetem) zur Platte gegossen. Bei den ersten Untersuchungen verwandten wir daneben auch Gelatineplatten; da wir hier jedoch nur eine grössere Zahl Schimmelpilze erhielten, wurde von der Gelatine Abstand genommen. Die folgende Tabelle enthält deshalb nur Zahlen, die sich aus der Zählung der Agarplatten und zwar 48 Stunden nach der Aussaat und Aufbewahrung im Brütschrank bei $37^{\circ} \mathrm{C}$. ergaben.

Tabelle .

\begin{tabular}{|c|c|c|c|c|}
\hline $\mathrm{Nr}$. & $\begin{array}{l}\text { Tag } \\
1898 \\
\end{array}$ & in Tagen & $\begin{array}{l}\mathrm{Nr} \text { der } \\
\text { Kälber }\end{array}$ & Zahl der Keime \\
\hline 1 & 4. April & 2 & $6 / 8$ & 8144000 \\
\hline 2 & 5. , , & 2 & $9 / 11$ & unzählig \\
\hline $\mathbf{3}$ & 28. , , & 2 & $28 / 31$ & 8064000 \\
\hline 4 & 30. , , & 13 & $12 / 14$ & 7024000 \\
\hline 5 & 30. , , & 1 & $36 / 39$ & unzählig \\
\hline 6 & 18. Mai & 14 & $9 / 11$ & 299500 , vgl. Nr. 2 \\
\hline 7 & 18. & 19 & $36 / 39$ & 1184500 , vgl. Nr. 5 \\
\hline 8 & 21. , , & 20 & $40 / 44$ & 2684300 \\
\hline 9 & 23. & 51 & $6 / 8$ & 207500 \\
\hline 10 & 23. , , & 51 & $9 / 11$ & 244250 \\
\hline 11 & $25 . \quad$, & 17 & $47 / 50$ & 1. 551000 \\
\hline 12 & 4. Juni & 20 & $59 / 62$ & 748500 \\
\hline 13 & 8. , & 23 & $63 / 66$ & 251750 \\
\hline 14 & 18. ., & 26 & $75 / 78$ & 116500 \\
\hline
\end{tabular}

Die Abnahme der Keimzahl zeigt also kein constantes Verhalten, wie Nr. 4 (13 Tage), Nr. 6 (14 Tage), Nr. 7 (19 Tage), Nr. 8 (20 Tage), Nr. 9 (51 Tage), Nr. 10 (51 Tage) u. A. bewiesen.

Was die Art der gefundenen Keime betrifft, so überwogen zuerst die Staphylokokken und zwar meist der albus, daneben fanden sich Kurz- und Langstäbchen, Sarcinen, bisweilen Staphylococcus aureus und auf den Gelatineplatten auch Schimmelpilze. Bei den letzten Proben fand sich fast ausschliesslich ein segmentirter Bacillus, der unter dem Mikroskop dem Diphtheriebacillus sehr ähnelte. Er zeigte dasselbe segmentirte Verhalten, lag häufig pallisadenartig und hatte bisweilen eine Anschwellung an dem einen Ende. Er ist unbeweglich, fürbt sich nach Gram, wächst auf Agar, Gelatine, ohne zu verflüssigen, Kartoffel, bildet anf der Bouillon ein Häutchen und bringt Milch zuerst zum Gerinnen, um sie nach einigen Tagen langsam zu verflüssigen. Charakteristisch ist sein Verbalten auf 
Batteriol. Erfahrungen Über die Königsberger ThierLymphe. 339

Agar: zuerst bildet er kleine, feuchtglänzende weisse Colonieen, die denen des Staphylococcus albus sehr ähneln, nach einigen Tagen wird die Oberfläche trocken und zeigt kleine Rinnen; in alten Culturen zeigt die Mitte dieses trockene, etwas gelbe Aussehen, während die Randpartieen grau und durchsichtig sind, so dass man eine Mischcultur vor sich zu haben glaubt. Dieser Bacillus fand sich sowohl in verschiedenen Proben von Lymphe, wie auch auf den Latten des Stallbodens, auf denen die Kälber lagen. Dass er sich in allen Proben der letzten Lymphe fand, erklärt sich daraus, dass zu den Impfungen der Kälber stets Lymphe vorher geimpfter Kälber verwandt wurde. In Nr. 7 war der Bacillus fast in Reincultur gefunden worden. Frr ist für weisse Mäuse und Meerschweinchen unschädlich, urd wie die Impfungen der Kinder ergaben, in der Lymphe offenbar auch für Mensehen.

Die Thierversuche ergaben die Unschädlichkeit unserer Lymphe für graue und weisse Mäuse, sowohl bei subcutaner wie bei intraperitonealer Impfung, und in einem Falle auch für Meerschweinchen. Von letzteren Thieren hatten wir, um das Thiermaterial zu schonen, nach dem ersten negativ ausgefallenen Versuche Abstand genommen. Die ersten Thierversuche wurden nach den Dreyer'schen Angaben gemacht: 1 Oese Lymphe wurde einer grauen Hausmaus subcutan beigebracht; ferner wurde je 1 mittelgrosse Oese Lymphe in $0.5 \mathrm{~cm}$ Peptonwasser einer grauen und dieselbe Dosis einer weissen MLaus intraperitoneal injicirt; drittens wurden 3 Oesen Lymphe in $1^{\mathrm{cm}}$ Peptonwasser einem Meerschweinchen ein Mal in die Bauchhöhle gespritzt. Später wurde zum Plattenausgiessen wie zu den Thierversuchen dieselbe Lösung von $0.1^{\mathrm{ccm}}$ Lymphe in $2.5^{\mathrm{ecm}}$ Bouillon verwandt, und zwar wurden je $0 \cdot 5^{\mathrm{cm}}$ dieser Mischung einer weissen bezw. grauen Maus intraperitoneal injicirt. In allen Versuchen blieben die Thiere ohne Krankheitserscheinungen am Leben.

Da auch die Probeimpfungen an Menschen im Lymphinstitut mit der noch frischen Lymphe keinerlei irgendwie bösartige Erscheinungen gezeigt hatten, darf man wohl die mitverimpften Bakterien als für den Menschen bedeutungslos bezeichnen.

Um jedoch dem ganzen Impfverfahren auch diejenigen Gefahren zu nehmen, die von einer Uebertragung von Keimen aus der Haut und den Kleidern der Impflinge in die Impfwunden herrühren könnten, wurde der Versuch gemacht, ein keimfreies Operationsfeld zu schaffen, dieses mit kejmfreien und keimabhaltenden Verbänden zu bedecken und bis zum Schluss der Wunde keimfrei zu erhalten. Selbstredend wurde mit sterilem Messer geimpft, und zwar wurde das Lindenborn'sche Platin-Iridiummesser hierfür wie für die verschiedenen Vorversuche benutzt. Zur Orientirung über die Zahl und Art der Keime, die bei den hiesigen Impflingen 
und Wiederimpflingen auf den betreffenden Theil des Oberarmes nach der zu Hause gesetzlich vorgeschriebenen Abwaschung noch vorkommen, sowie über die in dem „reinen" Hemd enthaltenen wurde mittels sterilen Wattetupfers - Diphtherietupfer, wie sie im hiesigen hygienischen Institut gebräuchlich sind - und sterilen Wassers a) die betreffende Partie des Oberarmes, b) der entsprechende Theil des Hemdes abgerieben und auf

\begin{tabular}{|l|l|}
\hline Ia. & Ib. \\
\hline
\end{tabular}
je $1 / 2$ Platte Glycerin-Agar in einzelnen Strichen abgezogen. Die Platte war durch einen gelben Strich in zwei Theile getheilt, links als Ia bezw. IIa u.s.w. bezeichnet, rechts als Ib, II b u.s.w., so dass a) die Keime von. der Haut, b) die vom Hemd enthielt. Zu dem am 12.IV. unternommenen Versuche wurden 5 Wiederimpflinge im hiesigen Lymphinstitnt verwandt. Es enthielten nach der gesetzlich vorgenommenen Waschung bezw. auf frischem Hemd die betreffenden Wiederimpflinge folgende Keime:

Ia. ca. 10 Colonicen von Staphyl. albus.

IIa. Ziemlich viele Colonieen von Staphylococcus albus.

III a. Zahlreiche Staphyl. albus.

IVa. Zahlreiche Staphyl. albus, vereinzelte Staphyl. aureus.

Va. Vielfach Staphyl. albus.
Ib. Vielfach Staphyl. albus, vereinzelte Colonie eines dicken, Gelatine und Agar verflüssigenden Bacillus.

II b. Wie II a.

III b. Wie IIIa.

IVb. Weniger zahlreiche Colonieen von Staphyl. albus.

Vb. Wie Va.

Dass lieine genaue Zahlenangabe gegeben ist, liegt daran, dass häufig lang gewachsene Colonieen sichtbar wurden, bei denen es nicht möglich war zu entscheiden, aus wie viel Keimen bezw. Einzelcolonieen sie bestanden.

Die zur Impfung verwandte Lymphe zeigte zahlreiche Colonieen von Staphylococcus albus und einige etwas gelber gefürbte Staphylokokken.

Die Ergebnisse der Impfung am 7. Tage waren:

I. 4 Pusteln, geringe Röthe um dieselben,

II. 4 Pusteln, jede mit einem etwa $1 \mathrm{~cm}$ breiten rothen Hof,

III. 2 Pusteln, wie sub II., aber etwas tiefere Röthe,

IV. 4 Pusteln, zusammengeflossene Röthung in der Ausdehnung von $5 \mathrm{~cm}$ Durchmesser,

V. 4 Pustelu, geringe blassrothe Fürbung. 
Bakteriol. Erfahrongen ÜBer DIE Köñgsberger Thierlymphe. 341

Dieser Versuch wurde am folgenden Tage, 13. IV., mit 5 Erstimpflingen wiederholt:

Ia. Nicht sehr zahlreiche St. albus und aureus, verschiedene $\mathrm{Co}$ lonieen von Kartoffelbacillus, einige von Sarcina lutea.

IIa. Dick besät mit St. albus, vereinzelte grosse Kokken (Luftkokken?).

III a. Dick besät mit St. albus.

IVa. Wenige St. albus und vereinzelte Colon. von fluorescirenden Bacillen: kürzere, zuweilen zu Fäden ausgewachsene Stäbchen, die Gelatine nicht verflüssigen, beweglich sind, auf Kartoffel mit gelbbraunem Belag wachsen, nach Gram negative Resultate geben, Milch nicht coaguliren, mit Chloroform keinen blauen Farbstoff geben, im Gelatinestich nur oberflächlich wachsen.

Ferner 2 Colonieen von RosaHefe, die jedoch auch aus der Luft stammen können, da sie erst ziemlich spät auf der Platte erschienen.

Va. Dick besät mit St. albus.

Ib. Wenige Colonieen von St. albus.

IIb. Wie IIa.

IIIb. Wie IIIa.

IVb. Einige St. albus, einige Sarcina lutea.

Die Lymphe ergab hauptsächlich Staphylococeus albus.

Die am 20. IV. vorgenommene Revision ergab 100 Procent personellen, wie Schnitterfolg und ganz geringe Röthe um die Pusteln herum.

Um den thatsächlichen Verhältnissen bei der Impfung möglichst gerecht zu werden, mussten aber auch die mehr in der Tiefe der Haut liegenden Keime untersucht werden. Zu diesem Zwecke wurden mit ausgeglühten Impfmessern die 4 Impfschnitte gemacht - die Haut war nicht desinficirt worden -, und die Schneide des Impfmessers auf umgekehrten Agarplatten ausgestrichen. Erst dann wurde nach nochmaliger Ausglühung des Messers Lymphe den Gläschen entnommen und in die bleine Wunde eingetragen.

18. IV. Es wurden 3 Erstimpflinge Nr. I bis III und 3 Wiederimpflinge Nr. IV bis VI verwandt. 
A. Erstimpflinge:

I. Spärliche Colonieen von Staphylococcus albus und aureus.

II. desgl.

III. desgl.

B. Wiederimpflinge:

IV. Spärliche Colonieen von Staphylococcus aureus und albus, eine grauweisse Colonie mit gezacktem Rand: kleine plumpe Bacillen mit zugespitzten Enden enthaltend.

V. Staphylococens aureus und albus, Sarcina lutea.

VI. reichliches Wachsthum von Staphylococcus aureus und albus, Kartoffelbacillus.

Das Impfresultat war trotzd́em ein gutes, ohne irgendwie beträchtliche Röthung oder Schwellung bei den Wiederimpflingen und absolute Reactionslosigkeit bei den Erstimpflingen.

Während diese Vorversuche in der hiesigen Königlichen Lymphgewinnungs-Austalt angestellt wurden, wo uns Hr. Director Dr. Luchau sein Material und seine Mithülfe freundlichst zur Verfügung stellte, wurden die eigentlichen, nun folgenden Versuche an den Impf- bezw. Wiederimpflingen der Kgl. medicinischen Poliklinik angestellt. Dem Leiter derselben, Hrn. Prof. Dr. Schreiber und seinen Assistenten gestatten wir uns auch an dieser Stelle ebenso wie Hrn. Dr. Luchau unseren verbindlichsten Dank auszusprechen.

Der erste Versuch wurde am 18.V. in der Weise angestellt, dass bei 2 Wiederimpflingen eine Desinfection der Haut mit neuer, ungebrauchter Handbürste, warmen sterilisirten Wasser und grüner Seife vorgenommen, darauf mehrfach mit 2 bis 3 sterilen Tupfern und 60 procent. Alkohol abgerieben wurde, darauf ebenso mit Sublimat, hierauf wieder mit Alkohol - im Ganzen etwa 5 Minuten. Das Verfahren ist schmerzhaft, die Haut wird stark geröthet - und was das Schlimmste ist, ergab keine guten Resultate. Gleichzeitig wurde 4 Erstimpflingen der entsprechende Theil des Oberarmes 2 Minuten lang mit öfters gewechselten sterilen Tupfern und 60 procent. Alkohol abgerieben; auch hier wurde die Haut stark geröthet; das Verfahren wurde jedoch nie bis zur Schmerzhaftigkeit getrieben.

Wiederum wurden jetzt mit ausgeglühtem Messer seichte Schnitte gemacht und auf Agarplatten diesmal nicht verrieben, sondern durch den Agar durchgezogen - eingeschnitten - in etwa 12 neben einander liegenden kurzen Schnitten. 
Batteriol. Erfahrungen Über die Köntgsberger Thierlymphe. 343

Das Resultat war folgendes:

A. Erstimpflinge.

I: 1 Keim, II : 0 Keime, III : 2 Keime, IV: 1 Keim.

B. Wiederimpflinge.

V: 7 Keime, VI: 4 Keime.

Es handelt sich um Staphylococcus albus und Sarcina lutea.

Nach Eintragung der Lymphe wurde der Verband über die Impf fläche gelegt, der ans einem kleinen Säckchen mehrfach zusammengelegter, mit Watte ausgestopfter Gaze bestand - sterilisirt - und mit zwei schmalen Heftpflasterstreifen fest an dem Arm befestigt.

Bei der am 25. V. vorgenommenen Revision ergab sich, dass die Erstimpflinge bei 100 Procent personellem Erfolg gar keine Reaction, die Wiederimpflinge beide ziemlich ausgedehnte Röthung des Oberarmes mit ebenfalls 100 Procent personellem Erfolg hatten. Nr. V hatte auch eine geringe Schwellung der Hatut.

Die Untersuchung der Lymphe (rergl. Tabelle I, Nr. 7) ergab 1184500 Colonieen, die aber fast nur jenen beschriebenen segmentirten Bacillus enthielten und nur vereinzelte Staphylokokken. Die geimpfte Maus blieb am Leben.

Ein Vergleich unserer Resultate mit dem der Massenimpfung mit derselben Lymphe, die an demselben Tage an 27 Lrst- und 30 Wiederimpflingen seitens der Poliklinik unternommen war, und wobei weder eine Desinfection der Haut noch ein Verband zur Anwendung kam, fiel zu Ungunsten des Desinfectionsverfahrens aus, indem von den Erstimpflingen einer eine unbedeutende Röthung in der näheren Umgebung der Pusteln zeigte; eins von 27 Kindern hatte keinen Impferfolg. Unter den 30 Wiederimpflingen hatten zwei einen etwas gerötheten und infiltrirten Oberarm. Die Impferfolge waren hier ungünstig, weil es sich fast ausschliesslich um zweite und dritte Wiederimpfungen handelte.

In Betreff unseres Verbandes bemerke ich, dass er nur bei einem unruhigen Kinde sich gelöst hatte. Es wurde nach der Besichtigung ein ebensolcher Verband auf den Oberarm gelegt und eine nochmalige Besichtigung nach einigen Tagen angeordnet, um auch über später auftretende Reactionen unterrichtet zu sein. Bemerkt sei gleich hier, dass bis auf einen unten zu beschreibenden Fall keine Reaction mehr sich zeigte, trotzdem alle Kinder noch ein zweites Mill - 14 Tage nach der Impfung - besichtigt wurden.

Am 21. V. wurde der Versuch wiederholt, diesmal wurde jedoch nur die Alkoholdesinfection angewandt. Es standen nur 1 Erst- und 2 Wiederimpflinge zur Verfügung. 
Die Impfschnitte auf Agar nach der Desinfection ergaben:

A. Erstimpf linge. I: 1 Keim.

B. Wiederimpflinge. II : 0 Keime, III : 0 Keime.

In Nr. I handelte es sich um ein kurzes, unbewegliches Stäbehen, lass sich nach Gram nicht färbte.

Die Lymphe hatte 2684300 Keime pro $1^{\text {ecm }}$ (vergl. Tabelle I, Nr. 8); es waren hauptsäehlich segmentirte Bacillen, vereinzelt Staphylococcus albus.

28. V. Nr. I hatte 3 Pusteln, Nr. II 4 Pusteln, -Nr. III 3 Pusteln, erstere beiden ohne jede Röthung, letztere mit geringẹ Röthung der Umgebung. Bei der Massenimpfung zeigte sich bei 31 Erstimpflingen $3 \mathrm{Mal}$ confluireude Röthe, bei 42 Wiederimptlingen $7 \mathrm{Mal}$ der Oberarm nehr oder minder weit geröthet, 2 Mal waren dabei Achseldrüsen geschwollen.

Am 25. V. wurde mit Lymphe von Kalb Nr. 47 bis 50 operirt. 2 Impflinge und 2 Wiederimpflinge wurden eirca 2 Minuten mit Alkohol abgewaschen, sonst wie am $21 . \mathrm{V}$.

Die vier angesetzten Platten zeigten nirgends einen Keim.

Die Lymphe enthielt 1551000 Keime pro $1^{\mathrm{cm}}$, die geimpfte Maus blieb am Leben. Unter den Keimen überwog bei Weitem der segmentirte Bacillus; ferner fand sich 1 Colonie weisse Sarcine und 1 Colonie eines schleimig-fidenziehenden Kapselbacillus.

Die Besichtigung unserer Kinder ergab am 2. VI. keinerlei Reactionserscheinungen, die der Massenimpfung unter 19 Erstimpflingen 3 Mal confluirende Röthe, und unter 39 Wiederimpflingen $5 \mathrm{Mal}$ einen gerötheten Oberarm.

28. V. Lymphe von Kalb Nr. 47 bis 50. Versuchsanordnung von jetzt ab stets wie am 25 . V.

2 Erstimpf linge, 2 Wiederimpflinge; nur am Arm des zweiten Wiederimpf linges konnten (nach der Alkobolabreibung) 9 Keime gezüchtet werden; 1 Colonie Staphylococcus albus, sonst kleine, der Pseudodiphtherie ähnliche Stäbchen. Die anderen Platten blieben keimfrei.

Dieser Wiederimpfling zeigte ebenso wie die Erstimpflinge keine Reaction, nur der andere Wiederimpfling hatte eine schwache Röthe um die Pusteln hernm.

Massenimpfung: 28 Erstimpflinge $2 \mathrm{Mal}$ confturende Röthe, 16 Wiederimpflinge $2 \mathrm{Mal}$ etwas grössere Röthung, durunter eine über den ganzen Oberarm.

4. VI. Lymphe von Kalb Nr. 59 bis 62 , enthält 748500 Keime pro $1 \mathrm{cem}$ meist segmentirter Bacillus, wenige Culonieen von Staphylococeus albus 
und eine aurens. 2 Impflinge, 2 Wiederimpflinge. Alle 4 Agarplatten keimfrei.

11. VI. Besichtigung: keine Reaction. - Die Massenimpfung ergab unter 25 Erstimpflingen $2 \mathrm{Mal}$ zusammenfliessende Röthe, unter 30 Wiederimpflingen $3 \mathrm{Mal}$ einen gerötheten Oberarm.

8. VI. Lymphe von Kalb Nr. 63 bis 66 , enthält 251750 Keime pro $1^{\mathrm{cem}}$; nur segmentirter Bacillus. 2 Impflinge, 2 Wiederimpflinge, Platten keimfrei. Besichtigung am 15. VI: keine Reaction. Massenimpfung: 29 Erstimpflinge mit confluirender Röthe, 23 Wiederimpflinge wobei 3 mit gerötheten, wenig geschwollenen Oberarmen.

11. VI. Lymphe wie am 8. VI. 2 Impflinge, bei Nr. II auf der Agarplatte: 1 Colonie mit grossen Luftkokken; 2 Wiederimpflinge, Agarplatten keimfrei, ebenso bei Erstimpfling Nr. I. Besichtigung am 18. VI: keine Reaction. Massenimpfung: 26 Erstimpflinge, 6 mit zusammenfliessender Röthe, 33 Wiederimpflinge, 5 mit zusammenfliessender Röthe.

15. VI. Lymphe wie am 8 . VI. 2 Impflinge, 2 Wiederimpflinge; Agarplatten keimfrei. Besichtigung am 22. VI: keine Reaction. Massenimpfung; 31 Erstimpflinge, 2 mit zusammenfliessender Röthe, 23 Wiederimpflinge, 3 mit zusammenfliessender Röthe.

18. VI. Lymphe von Kalb Nr. 74 bis 78 , enthält 116500 Keime pro $1 \mathrm{cem}$, nur segmentrirter Bacillus. 1 Erstimpfling, 2. Wiederimpflinge. Agarplatten keimfrei. Bei der Besichtigung am 25. VI. zeigte Wiederimpfling Nr. I Röthung und Schwellung des Oberarmes, die beiden anderen Kinder hatten keine Reaictionserscheinungen. Die Massenimpfung ergab unter 37 Erstimpflingen $3 \mathrm{Mal}$, unter 26 Wiederimpflingen 6 Mal geringe Röthung der Pustelumgebung.

Um über noch grössere Zahlen zu verfügen, wurden von Hrn. Dr. Luchau unter unserer Assistenz 122 Knaben (Wiederimpflinge) nach Alkoholabreibung, und gleich darauf 110 Mädchen (ebenfalls Wiederimpflinge) ohne Alkoholabreibung mit Lymphe von Kalb Nr. 74 bis 78 (vgl. 18. VI.) geimpft. Die erstere ergab 16-, die letztere 22 Mal zusammenfliessende Röthe mit geringer Schwellung der Haut des Oberarmes.

Um die Resultate vergleichen zu können, muss hier noch nachgetragen werden, dass am 17. VI. der eine Impfling, der am 21. V. von uns mit Alkoholabreibung und Verbänden behandelt war, in die Poliklinik gebracht wurde mit einem etwa haselnussgrossen Abscess, der eröffnet wurde und eine Reincultur von Staphyl. albus aufwies. Das Kind soll etwa 15 Tage nach der Impfung eine Schwellung des Oberarmes bekommen haben, die nach Aussage der Mutter durch Auflegen von Vaselineläppchen beseitigt wurde. Indessen heilten die Pusteln nicht zu, und es entstand allmählich jener Abscess, der nach einfacher Eröffnnng anstandslos 
heilte. Es muss noch bemerkt werden, dass die beiden Verbände - der eine vom Tage der Impfung bis zum 8. Tage, der andere vom 8 . bis 14. Tage gut gehalten, aber. doch die Anschwellung nicht verhindert hatten: Es scheint hier eine bestimmte Disposition vorzuliegen, die auch sonst beobachtet wird, wobei die Pusteln keine oder nur geringe Heilungstendenz zeigen und dabei Luft- oder Hautkeimen Gelegenheit zu secundären Infectionen geben. Noch am 17. VI. waren zwei Pusteln relativ gross und eitrig belegt. Später heilten sie.

Abgesehen von diesem Falle zeigten sich bei allen Impfungen keinerlei irgendwie erhebliche Reactionen.

Ein Vergleich zwischen unserem Verfabren (Alkoholabreibung und zwei Mal gewechselte sterile Verbände) ergiebt auf 18 Erstimpflinge keine, auf 18 Wiederimpflinge 5 Reactionen, denen die Erfolge der Poliklinik (einfache Hautwaschung, keinerlei Verband) mit 17 Reactionen unter 253 Erstimpflingen und 35 Reactionen unter 262 Wiederimpflingen gegenüberstehen. In Procenten ausgedrückt bedeutet dies bei Erstimpflingen 0 Proc. Reaction bei uns, gegen 6 -6 Proc. der Poliklinik, bei Wiederimpflingen 27.7 Proc. gegen 13.3 Proc. Und will man sämmtliche Erfolge der Alkoholabreibung ersehen und dazu die 122 Knaben gegenüber den 110 nicht mit Alkohol abgeriebenen Mädchen, vgl. 15. VI., mit in die Berechnung einschliessen, so ergeben sich, da man nur Wiederimpflinge unter sich vergleichen darf, bei 140 mit Alkohol behandelten 21 Reactionen, bei 372 nicht mit Alkohol behandelten 57 Reactionen, d. h. 15 Procent gegen 15 Procent. Ein Erfolg der Alkoholabreibung ist nicht zu erkennen, ebenso wenig ein Nutzen der sterilen Verbände.

Dagegen war namentlich bei der Massenimpfung am 15. VI. deutlich zu sehen, dass die mit Alkohol behandelten Kinder geringere Pustelentwickelung zeigten, sowohl was die Zahl, als auch was die Grösse der Pusteln betrifft. Hierfür mag folgendes Vorkommniss eine Erklärung geben: Anna Werbunat, am 11. VI. nach Alkoholabreibung geimpft und mit sterilem Verband versehen, zeigte am 18. VI. keine Pustel. Sie wurde sofort noch einmal geimpft und es entwickelte sich bis zum 25. VI. eine Pustel. Dies ist nur dadurch zu erklären, dass aus der Tiefe der Hautporen der eingeriebene Alkohol nicht genügend verdunsten kann und hier keimabtödtend wirkt. Für diese Auffassung spricht auch der Umstand, dass bei unseren Versuchen, wo wir die Haut energisch mit Alkohol abgerieben hatten, eine Weile bis zur Trocknung der Haut warteten, dann mit sterilem Messer kleine Schnitte in die Haut machten und das Messer auf Agar verrieben oder einschnitten, fast immer Keimfreiheit der Agarplatten erfolgte. Offenbar gelangten hier kleinste Tröpfehen aus der Tiefe der 


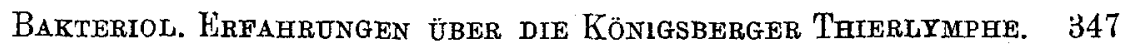

Hautporen mit dem Messer und dem Gewebssaft auf den Agar und verhinderten ein Aufgehen von etwa mit übertragenen Keimen.

Wir haben stets mehrere Minuten gewartet, bis der Alkohol von der Hautoberfläche verdunstet war, bevor wir die Schnitte machten. - Dass nicht nur weniger, sondern auch kleinere Pusteln bei der Alkoholabreibung aufgingen, ist einleuchtend, wenn man sich die Koch'sche Erklärung der Pusteln vorhält, wonach, wie ja auch der Augenschein beweist, jede grössere Pustel aus mehreren kleineren besteht. 